\title{
Lane-deviation penalty formulation and analysis for autonomous vehicle avoidance maneuvers
}

Pavel Anistratov, Björn Olofsson and Lars Nielsen

The self-archived postprint version of this journal article is available at Linköping University Institutional Repository (DiVA):

http://urn.kb.se/resolve?urn=urn:nbn:se:liu:diva-178560

N.B.: When citing this work, cite the original publication.

Anistratov, P., Olofsson, B., Nielsen, L., (2021), Lane-deviation penalty formulation and analysis for autonomous vehicle avoidance maneuvers, Proceedings of the Institution of mechanical engineers. Part D, journal of automobile engineering, 235(12), 3036-3050.

https://doi.org/10.1177/09544070211007979

Original publication available at:

https://doi.org/10.1177/09544070211007979

Copyright: Institution of Engineering and Technology (IET)

http://www.theiet.org/ 


\title{
Lane-Deviation Penalty Formulation and Analysis for Autonomous Vehicle Avoidance Maneuvers
}

\author{
Pavel Anistratov ${ }^{1}$, Björn Olofsson ${ }^{1,2}$, and Lars Nielsen ${ }^{1}$
}

\begin{abstract}
Autonomous vehicles hold promise for increased vehicle and traffic safety, and there are several developments in the field where one example is an avoidance maneuver. There it is dangerous for the vehicle to be in the opposing lane, but it is safe to drive in the original lane again after the obstacle. To capture this basic observation, a lane-deviation penalty (LDP) objective function is devised. Based on this objective function, a formulation is developed utilizing optimal allwheel braking and steering at the limit of road-tire friction. This method is evaluated for a double lane-change scenario by computing the resulting behavior for several interesting cases, where parameters of the emergency situation such as the initial speed of the vehicle and the size and placement of the obstacle are varied, and it performs well. A comparison with maneuvers obtained by minimum-time and other lateral-penalty objective functions shows that the use of the considered penalty function decreases the time that the vehicle spends in the opposing lane.
\end{abstract}

\section{Keywords}

Active safety systems, vehicle control systems, intelligent vehicles, vehicle dynamics, passenger vehicles, at-the-limit operation, double lane change.

\section{Introduction}

Much of the development within autonomous driving and autonomous vehicles is discussed in terms of the standard SAE J3016 ${ }^{1}$, and the levels of autonomy defined there. When driving a car, if the driver is required to be actively involved in that task, and to be ready to take over when asked to, then such a car is not a self-driving car according to the National Transportation Safety Board ${ }^{2}$. Thus, to reach the higher levels of autonomous driving, it is necessary that the vehicle is able to handle critical situations at the limit of road-tire friction, and it is therefore natural that this is an active area of research ${ }^{3-6}$, and it has a great potential to save lives by avoiding or mitigating severe traffic accidents ${ }^{7}$. One example of a situation with a risk of passenger fatality and severe injuries, is when an obstacle more or less suddenly appears in front of the vehicle. Then it is necessary to perform a maneuver which in Scandinavian countries is known as the "moose test", see Figure 1. Technically this is a double lanechange maneuver for which there is a standardized version, the ISO 3888-2 double lane-change test ${ }^{8}$.

Obstacle avoidance and safety maneuvers have been proposed previously, see ${ }^{9-11}$ for some examples and Section "Related Research" for more details. Generally speaking, the task is to fully utilize the available controls, that is, the steering angle and the braking/driving torques on the wheels. With emerging advanced sensor platforms for situation awareness, the problem can be formulated as an optimal control problem with the scenario constraints included in the optimization ${ }^{12}$. When it comes to the optimization criterion, there are earlier suggestions, for example, using criteria such as minimum time of the maneuver ${ }^{12}$ or a weighted sum of the entry and exit speeds ${ }^{6}$. The criterion used here is different from those, and it is based on the observation that

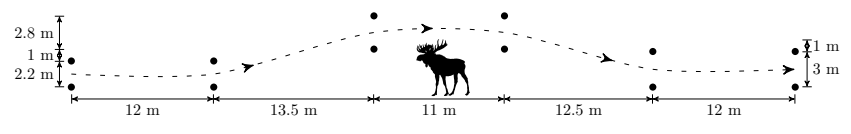

Figure 1. Sketch of the double lane-change maneuver with a potential obstacle.

it is dangerous for the vehicle to be in the opposing lane, but it is safe when the vehicle is back in its original lane. To capture this fundamental idea, a lane-deviation penalty (LDP) objective function is devised where being outside the own lane is penalized. A preliminary version of this idea and its formulation using a single-track vehicle model was presented in our conference contribution ${ }^{13}$. The LDP has also been one of the criteria used when developing recovery behavior after an avoidance maneuver ${ }^{14}$.

\section{Contributions}

A major contribution of the now presented research is a careful and thorough treatment and analysis of the LDP formulation, and the formulation is shown to work well by giving efficient and stable maneuvers. Using a double-track vehicle model with load transfer to analyze the performance is new compared to initial studies ${ }^{13}$. Further, the behavior is illustrated in several scenarios varying parameters like

\footnotetext{
${ }^{1}$ Division of Vehicular Systems, Department of Electrical Engineering, Linköping University, Sweden

${ }^{2}$ Department of Automatic Control, Lund University, Sweden

\section{Corresponding author:}

Pavel Anistratov, Department of Electrical Engineering, Linköping University, Linköping, SE-581 83, Sweden

Email: pavel.anistratov@liu.se
} 
distance to the obstacle and size of the obstacle. Additionally, the studied velocities range from the low end, where it may be possible to do an emergency braking, to the high end, where the speed is on the border of being too high to handle the obstacle satisfactorily. An additional contribution is the result from the comparison with minimum-time formulations and two other lateral-penalty formulations, where it in fact turns out that the LDP, being a penalty on position, nevertheless results in less time in the opposing lane than the minimum-time formulation for the complete maneuver.

\section{Related Research}

Optimization has proven fruitful in understanding and designing safety systems, and examples in the literature include: a study of at-the-limit maneuvers and a comparison of vehicle models of different complexity used in optimizations $^{12}$; an analysis of the minimum distance when an avoidance maneuver is still possible ${ }^{9}$; utilization of an optimal-control based method for quantifying the maneuverability of autonomous vehicles during emergency highway-speed situations ${ }^{11}$; and a study on how to split a full maneuver into maneuver segments ${ }^{15}$.

One line of optimal-control approaches uses a simplified vehicle model to obtain reference values for the underlying control system, for example, for performing an avoidance maneuver by applying the modified Hamiltonian algorithm for nonlinear optimal control allocation ${ }^{16}$, for preventing road departure on curved roads ${ }^{17}$, or for assisting in collision mitigation with oncoming vehicles ${ }^{18}$. A related approach is to reformulate optimal control problems in a modelpredictive control fashion, which has been successfully applied, for example, for high-speed lane changes ${ }^{19}$, to move on curvy roads with replanning ${ }^{3,20}$, for obstacle avoidance by representing the vehicle with a set of circles ${ }^{4}$, and in a study of four-wheel-independent vehicles ${ }^{21}$.

Motion planning is also possible to approach by considering an artificial potential field to guide the vehicle ${ }^{10}$. This approach has been experimentally validated for lanekeeping driver assistance ${ }^{22}$. The idea has been extended by including model predictive control applied after the path has been computed using the defined artificial potential field ${ }^{23}$.

To improve the overall behavior of the vehicle, different approaches have been suggested where the control scheme takes into account, for example, changing tire stiffness ${ }^{24}$, varying traction limitations ${ }^{5}$, uncertain mass of the vehicle $^{25}$, adaptation of the level of braking ${ }^{26}$, or different criteria for different phases of the maneuver ${ }^{11}$.

\section{Outline}

The outline of the paper is to first present models of vehicle, obstacle, and road, and then to formulate an optimal control problem representing the motion-planning problem. Then follow in order the results on scenario variations for different initial velocities and obstacles with discussion on overall motion planning, a comparison with other criteria, a discussion, and conclusions.

\section{Modeling}

This section presents the equations for the considered vehicle model, limits on the model variables as a result of physical

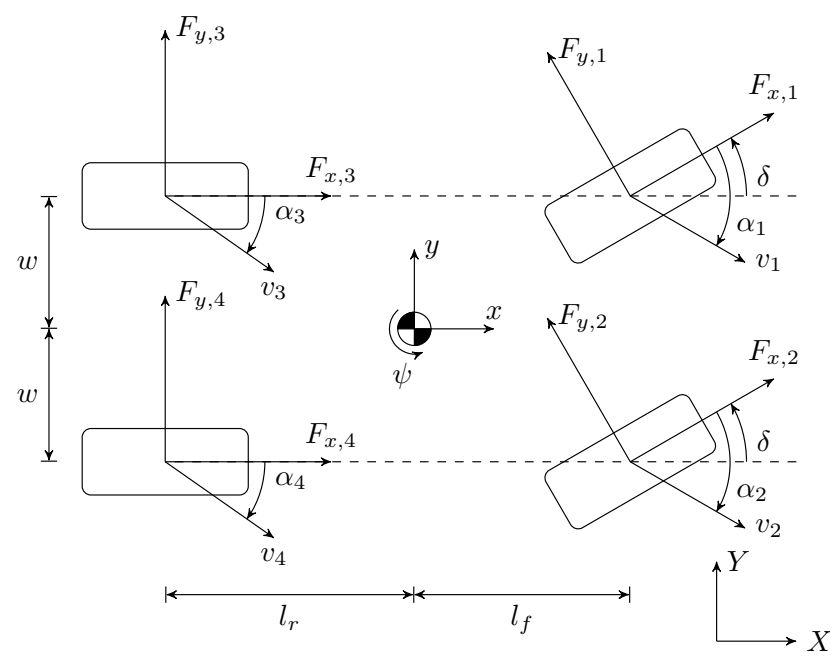

Figure 2. The double-track model used for vehicle modeling.

constraints, and specifies the road configuration for the investigated double lane-change maneuver.

\section{Chassis Model}

The double-track chassis model ${ }^{27}$ with accounted pitch and roll dynamics is used to describe the vehicle with the following equations of motion:

$$
\begin{aligned}
\dot{v}_{x}-v_{y} \dot{\psi}-h f_{\theta}(\theta, \dot{\theta}, \ddot{\theta}, \phi, \dot{\phi}, \ddot{\phi}, \dot{\psi}, \ddot{\psi}) & =\frac{F_{X}}{m}, \\
\dot{v}_{y}+v_{x} \dot{\psi}-h f_{\phi}(\theta, \dot{\theta}, \ddot{\theta}, \phi, \dot{\phi}, \ddot{\phi}, \dot{\psi}, \ddot{\psi}) & =\frac{F_{Y}}{m} \\
\ddot{\psi} I_{\psi}\left(\theta, \phi, I_{B}\right)+h f_{\psi}\left(\theta, \phi, F_{X}, F_{Y}\right) & =M_{Z},
\end{aligned}
$$

where $v_{x}, v_{y}$ are the longitudinal and lateral velocities at the center of mass, $\dot{\psi}$ is the yaw rate, $h$ is the distance from the roll center to the center of mass, and $m$ is the vehicle mass. The functions $f_{\theta}(\cdot)$ and $f_{\phi}(\cdot)$ represent the contributions from the pitch and roll dynamics, respectively, where $\theta$ is the pitch angle and $\phi$ is the roll angle. These functions are introduced to the purpose of compact presentation, and they are together with the definition of other expressions in this section given in full in Appendix A. The moment of inertia associated with yaw angle $\psi$ is denoted by $I_{\psi}(\cdot)$, $I_{B}$ compactly denotes the vehicle chassis inertias $I_{x x}, I_{y y}$, and $I_{z z}$ along the three directions, and $f_{\psi}(\cdot)$ represents an additional contribution to the yaw dynamics from the deflection of the center of mass of the chassis. The global forces are denoted by $F_{X}, F_{Y}$, and $M_{Z}$ and are dependent on the longitudinal and lateral forces on all wheels, $F_{x, i}$, $F_{y, i}, i \in\{1,2,3,4\}$, and the steering angle $\delta$ (see Appendix A). To sum up, the pitch and roll dynamics are thus:

$$
\begin{gathered}
\ddot{\theta} I_{\theta}\left(\phi, I_{B}\right)=h f_{\theta, 1}\left(\theta, \psi, F_{X}\right)+f_{\theta, 2}\left(\theta, \dot{\theta}, \phi, \dot{\phi}, \dot{\psi}, I_{B}\right), \\
\ddot{\phi} I_{\phi}\left(\theta, \phi, I_{B}\right)=h f_{\phi, 1}\left(\theta, \phi, F_{Y}\right)+f_{\phi, 2}\left(\theta, \dot{\theta}, \phi, \dot{\phi}, \dot{\psi}, I_{B}\right) .
\end{gathered}
$$

The longitudinal load-transfer system is modeled as a rotational spring and damper system. By letting $K_{\theta}$ and $D_{\theta}$ be the rotational spring and damping parameters in the $\theta$-direction, the equations of the longitudinal load transfer 
Table 1. The vehicle model parameters. The chassis and wheel parameters ${ }^{12}$ and the tire-model parameters ${ }^{28}$ are adopted.

(a) Chassis and wheel.

\begin{tabular}{cc|cc}
\hline Notation & Value & Notation & Value \\
\hline$l_{f}$ & $1.3 \mathrm{~m}$ & $I_{x x}$ & $765 \mathrm{kgm}^{2}$ \\
$l_{r}$ & $1.5 \mathrm{~m}$ & $I_{y y}$ & $3477 \mathrm{kgm}^{2}$ \\
$w$ & $0.8 \mathrm{~m}$ & $I_{z z}$ & $3900 \mathrm{kgm}^{2}$ \\
$h$ & $0.5 \mathrm{~m}$ & $g$ & $9.82 \mathrm{~ms}^{-2}$ \\
$m$ & $2100 \mathrm{~kg}$ & $K_{\phi, f}, K_{\phi, r}$ & $89000 \mathrm{Nm}(\mathrm{rad})^{-1}$ \\
$R_{\omega}$ & $0.3 \mathrm{~m}$ & $D_{\phi, f}, D_{\phi, r}$ & $8000 \mathrm{Nms}(\mathrm{rad})^{-1}$ \\
$I_{\omega}$ & $4.0 \mathrm{kgm}^{2}$ & $K_{\theta}$ & $363540 \mathrm{Nm}(\mathrm{rad})^{-1}$ \\
$\sigma$ & $0.3 \mathrm{~m}$ & $D_{\theta}$ & $30960 \mathrm{Nms}(\mathrm{rad})^{-1}$ \\
\hline
\end{tabular}

(b) Tire (dry asphalt). The same parameters are used for both the left and right wheels on each axle.

\begin{tabular}{ccc|ccc}
\hline Notation & Front & Rear & Notation & Front & Rear \\
\hline$\mu_{x}$ & 1.2 & 1.2 & $E_{y}$ & -1.21 & -1.11 \\
$B_{x}$ & 11.7 & 11.1 & $B_{x 1}$ & 12.4 & 12.4 \\
$C_{x}$ & 1.69 & 1.69 & $B_{x 2}$ & -10.8 & -10.8 \\
$E_{x}$ & 0.377 & 0.362 & $C_{x \alpha}$ & 1.09 & 1.09 \\
$\mu_{y}$ & 0.935 & 0.961 & $B_{y 1}$ & 6.46 & 6.46 \\
$B_{y}$ & 8.86 & 9.3 & $B_{y 2}$ & 4.20 & 4.20 \\
$C_{y}$ & 1.19 & 1.19 & $C_{y \kappa}$ & 1.08 & 1.08 \\
\hline
\end{tabular}

$\operatorname{are}^{12}$ :

$$
\begin{aligned}
\left(F_{z, 1}+F_{z, 2}\right) l_{f}-\left(F_{z, 3}+F_{z, 4}\right) l_{r} & =K_{\theta} \theta+D_{\theta} \dot{\theta} \\
F_{z, 1}+F_{z, 2}+F_{z, 3}+F_{z, 4} & =m g
\end{aligned}
$$

where $F_{z, i}, i \in\{1,2,3,4\}$, denote normal forces, $g$ is the constant of gravitational acceleration, and $l_{f}$ and $l_{r}$ are defined in Figure 2. Using $K_{\phi, f}$ and $D_{\phi, f}$ for the rotational spring and damping parameters for the front axle in the $\phi$ direction, and using $K_{\phi, r}$ and $D_{\phi, r}$ analogously for the rear axle, the lateral load transfer is determined by Berntorp et al. ${ }^{12}$ :

$$
\begin{aligned}
& -w\left(F_{z, 1}-F_{z, 2}\right)=K_{\phi, f} \phi+D_{\phi, f} \dot{\phi}, \\
& -w\left(F_{z, 3}-F_{z, 4}\right)=K_{\phi, r} \phi+D_{\phi, r} \dot{\phi},
\end{aligned}
$$

where $w$ is defined in Figure 2. The numerical values of the chassis parameters are given in Table 1a. To reflect the actual physical limitations of the vehicle inputs in the model, the absolute value of the steering angle and its first derivative are limited by the values $\delta_{\max }=0.5 \mathrm{rad}$ and $\dot{\delta}_{\max }=1 \mathrm{rad} / \mathrm{s}$, respectively.

The global vehicle position in $X$ and $Y$ coordinates (see Figure 2) is computed by integration of:

$$
\begin{aligned}
\dot{X} & =v_{x} \cos (\psi)-v_{y} \sin (\psi), \\
\dot{Y} & =v_{x} \sin (\psi)+v_{y} \cos (\psi),
\end{aligned}
$$

where $\psi$ is the heading angle of the vehicle relative to the $X$-axis in the global $X Y$-frame.

\section{Wheel Dynamics}

The dynamic equations for the wheels ${ }^{12}$ are given by

$$
T_{i}-I_{\omega} \dot{\omega}_{i}-F_{x, i} R_{\omega}=0, i \in\{1,2,3,4\},
$$

where $R_{\omega}$ is the wheel radius, $T_{i}$ is the driving/braking torque, and $I_{\omega}$ is the wheel inertia. The lower and upper limits on the wheel torque are chosen as

$$
\begin{aligned}
T_{i, \min } & =-\mu_{x, i} R_{\omega} m g, T_{1, \max }=T_{2, \max }=0, \\
T_{3, \max } & =T_{4, \max }=\mu_{x, i} R_{\omega} F_{z 0, r},
\end{aligned}
$$

where $i \in\{1,2,3,4\}, \mu_{x, i}$ are friction coefficients, and the rear nominal normal force $F_{z 0, r}$ is

$$
F_{z 0, r}=m g \frac{l_{f}}{l_{f}+l_{r}} .
$$

The limits on the first derivative of the wheel torque are chosen as ${ }^{12}$

$$
\dot{T}_{i, \max }=2.5 \mu_{x, i} R_{\omega} m g, i \in\{1,2,3,4\} .
$$

Numerical values for the parameters of the wheel dynamics are given in Table 1.

\section{Tire Model}

The slip angles ${ }^{28} \alpha_{i}$ and the slip ratios ${ }^{28} \kappa_{i}$ are defined as

$$
\begin{gathered}
\dot{\alpha}_{i} \frac{\sigma}{v_{x, i}}+\alpha_{i}=-\arctan \left(\frac{v_{y, i}}{v_{x, i}}\right), \\
\kappa_{i}=\frac{R_{\omega} \omega_{i}-v_{x, i}}{v_{x, i}}, i \in\{1,2,3,4\},
\end{gathered}
$$

where $\sigma$ is the relaxation length, $\omega_{i}$ is the angular velocity for wheel $i, v_{x, i}$ and $v_{y, i}$ are the longitudinal and lateral velocities for wheel $i$, with respect to its own coordinate frame.

The nominal tire forces $F_{x 0, i}$ and $F_{y 0, i}$ are computed using the Pacejka's Magic Formula model ${ }^{28}$ according to

$$
\begin{aligned}
F_{x 0, i}= & \mu_{x, i} F_{z, i} \sin \left(C _ { x , i } \operatorname { a r c t a n } \left(B_{x, i} \kappa_{i}\right.\right. \\
& \left.-E_{x, i}\left(B_{x, i} \kappa_{i}-\arctan \left(B_{x, i} \kappa_{i}\right)\right)\right), \\
F_{y 0, i}= & \mu_{y, i} F_{z, i} \sin \left(C _ { y , i } \operatorname { a r c t a n } \left(B_{y, i} \alpha_{i}\right.\right. \\
& \left.\left.-E_{y, i}\left(B_{y, i} \alpha_{i}-\arctan \left(B_{y, i} \alpha_{i}\right)\right)\right)\right),
\end{aligned}
$$

for each wheel $i \in\{1,2,3,4\}$, where $\mu_{x}$ and $\mu_{y}$ are the friction coefficients and $B, C$, and $E$ are model parameters (which are wheel dependent). The forces under combined longitudinal and lateral slip are calculated using weighting functions ${ }^{28} G_{x \alpha, i}$ and $G_{y \kappa, i}$ for the longitudinal and the lateral directions:

$$
\begin{aligned}
H_{x \alpha, i} & =B_{x 1, i} \cos \left(\arctan \left(B_{x 2, i} \kappa_{i}\right)\right), \\
G_{x \alpha, i} & =\cos \left(C_{x \alpha, i} \arctan \left(H_{x \alpha, i} \alpha_{i}\right)\right), \\
F_{x, i} & =F_{x 0, i} G_{x \alpha, i}, \\
H_{y \kappa, i} & =B_{y 1, i} \cos \left(\arctan \left(B_{y 2, i} \alpha_{i}\right)\right), \\
G_{y \kappa, i} & =\cos \left(C_{y \kappa, i} \arctan \left(H_{y \kappa, i} \kappa_{i}\right)\right), \\
F_{y, i} & =F_{y 0, i} G_{y \kappa, i}, i \in\{1,2,3,4\},
\end{aligned}
$$

where $B$ and $C$ are model parameters (which are wheel dependent). Numerical values of the tire parameters representing the behavior on dry asphalt ${ }^{28}$ are adopted and shown in Table $1 \mathrm{~b}$. The resulting tire forces with the numerical parameters in Table $1 \mathrm{~b}$ for the rear left wheel are visualized in Figure 3 for the cases of pure slip and combined slip. 


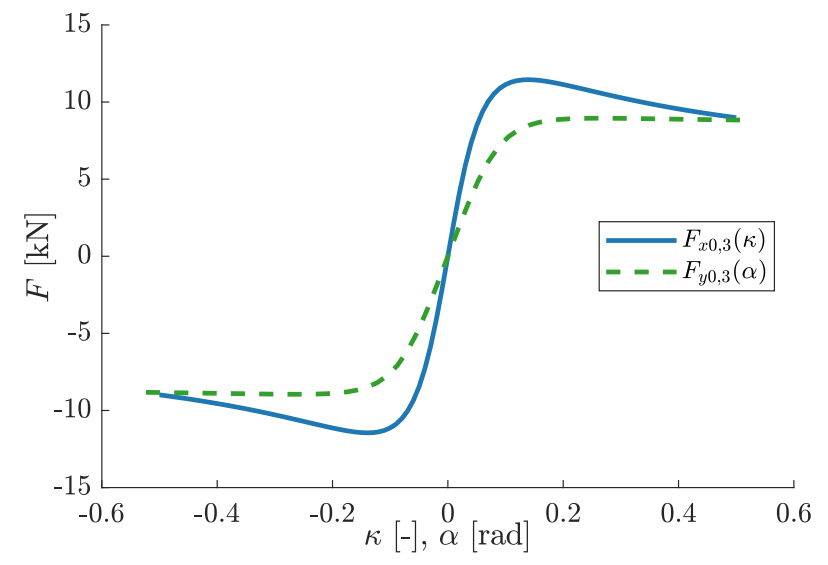

(a) Longitudinal and lateral forces depending on slip values for the case of pure slip.

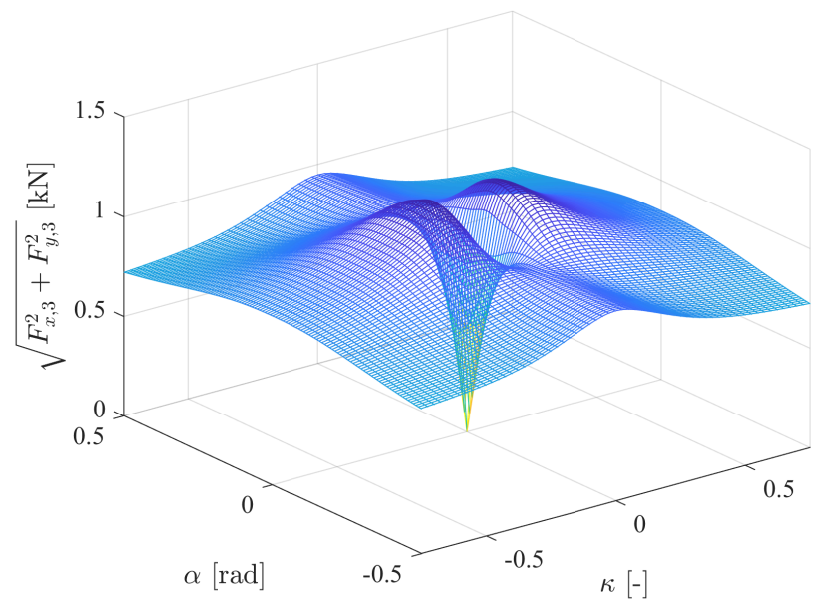

(b) Resulting tire-force surface for the case of combined longitudinal and lateral slip.

Figure 3. Tire forces for the left rear wheel with model parameters according to Table $1 \mathrm{~b}$.

\section{Summary of Vehicle Model}

To summarize the model equations (1)-(26), the input, state, and algebraic variables are collected in vectors. The time derivatives of the wheel torques and the steering angle are considered as inputs (this is beneficial in the optimization since it allows constraints also on the derivatives of the actual inputs)

$$
\mathbf{u}=\left\{\dot{\delta}, \dot{T}_{1}, \dot{T}_{2}, \dot{T}_{3}, \dot{T}_{4}\right\}
$$

The state vector is given by

$$
\begin{array}{r}
\mathbf{x}=\left\{X, Y, \psi, \dot{\psi}, T_{1}, T_{2}, T_{3}, T_{4}, \delta, v_{x}, v_{y}\right. \\
\left.\omega_{1}, \omega_{2}, \omega_{3}, \omega_{4}, \alpha_{1}, \alpha_{2}, \alpha_{3}, \alpha_{4}\right\} .
\end{array}
$$

Finally, the algebraic variables are combined into the vector

$$
\begin{array}{r}
\mathbf{z}=\left\{F_{X}, F_{Y}, M_{Z}, F_{x, 1}, F_{x, 2}, F_{x, 3}, F_{x, 4}, F_{y, 1}, F_{y, 2},\right. \\
F_{y, 3}, F_{y, 4}, v_{x, 1}, v_{x, 2}, v_{x, 3}, v_{x, 4}, v_{y, 1}, v_{y, 2}, v_{y, 3}, v_{y, 4}, \\
\kappa_{1}, \kappa_{2}, \kappa_{3}, \kappa_{4}, F_{x 0,1}, F_{x 0,2}, F_{x 0,3}, F_{x 0,4}, F_{y 0,1}, F_{y 0,2}, \\
F_{y 0,3}, F_{y 0,4}, F_{z, 1}, F_{z, 2}, F_{z, 4}, F_{z, 4}, H_{x \alpha, 1}, H_{x \alpha, 2}, \\
H_{x \alpha, 3}, H_{x \alpha, 4}, G_{x \alpha, 1}, G_{x \alpha, 2}, G_{x \alpha, 3}, G_{x \alpha, 4}, H_{y \kappa, 1}, \\
\left.H_{y \kappa, 2}, H_{y \kappa, 3}, H_{y \kappa, 4}, G_{y \kappa, 1}, G_{y \kappa, 2}, G_{y \kappa, 3}, G_{y \kappa, 4}\right\} .
\end{array}
$$

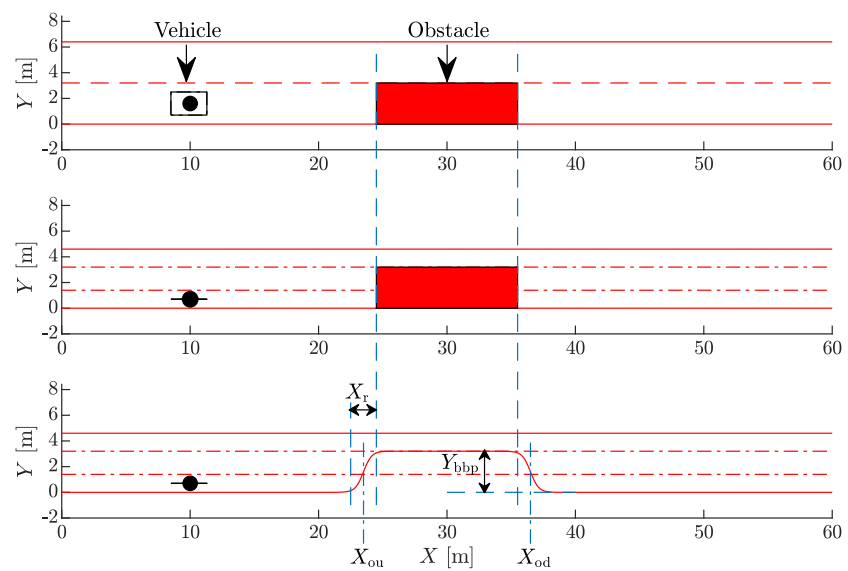

Figure 4. Graphical illustration of the road representation. The center of mass of the vehicle is shown as a circle. The top plot shows the actual road dimensions; the vehicle size is shown with the box. The middle plot shows the adjusted road dimensions with the vehicle represented only with its center of mass. The bottom plot shows the obstacle representation using (33) with parameters for the obstacle according to Table 2.

By using the defined vectors of input, state, and algebraic variables, a compact representation of the vehicle model ((1)-(26) including the expressions in Appendix A) can be established as

$$
\begin{aligned}
\dot{\mathbf{x}} & =G(\mathbf{x}, \mathbf{z}, \mathbf{u}), \\
0 & =h(\mathbf{x}, \mathbf{z}, \mathbf{u}),
\end{aligned}
$$

where the dynamic equations (some examples are (1)(3), (10)-(12)) are represented by (30) and the algebraic equations (here examples are (19)-(20)) are described by (31). The vehicle model is implemented using the declarative modeling language Modelica ${ }^{29}$.

\section{Modeling of Road and Obstacle}

The scenario considered in this paper is an avoidance maneuver for a vehicle driving on a two-lane road with one lane for traffic in each direction. In this scenario, an obstacle appears on the road, which is blocking the entire driving lane of the vehicle. The dimensions of the setup are shown in Figure 4. The top plot shows the actual dimensions of the road and the vehicle. In the optimization formulation, constraints are enforced only on the center of mass of the vehicle and therefore the road constraints are adjusted accordingly to consider the width of the vehicle as illustrated in the middle plot in Figure 4.

The driving boundaries are inspired by the ISO 38882 standard $^{8}$ and are formulated in an analytical form using a function $\widetilde{H}_{a_{\mathrm{o}}}^{a_{\mathrm{r}}}(a)$. This function represents a smooth approximation of the Heaviside step function with an offset $a_{\mathrm{o}}$ and a rising distance $a_{\mathrm{r}}$, the function is also known as the sigmoid function (see Figure 5)

$$
\widetilde{H}_{a_{\mathrm{o}}}^{a_{\mathrm{r}}}(a)=\frac{1}{2}+\frac{1}{2} \tanh \left(\frac{\pi}{a_{\mathrm{r}}}\left(a-a_{\mathrm{o}}\right)\right) .
$$

The analytical form of the right-hand side driving boundary ${ }^{13}$ is introduced as

$$
Y_{\mathrm{bb}}(X(t))=Y_{\mathrm{bbp}}\left(\widetilde{H}_{X_{\mathrm{ou}}}^{X_{\mathrm{r}}}(X(t))-\widetilde{H}_{X_{\mathrm{od}}}^{X_{\mathrm{r}}}(X(t))\right),
$$




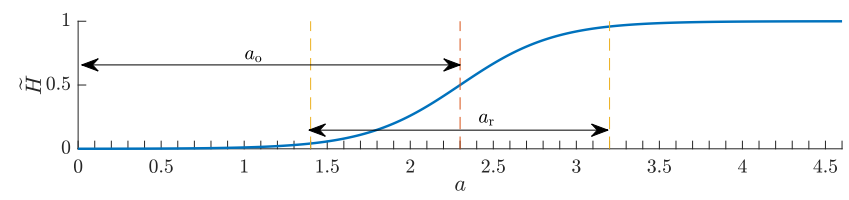

Figure 5. Illustration of the function $\widetilde{H}$ for the parameters $a_{\mathrm{o}}=2.3$ and $a_{\mathrm{r}}=1.8$.

Table 2. Nominal parameters defining the obstacle and the considered scenario.

\begin{tabular}{ccc|ccc}
\hline Notation & Value & Unit & Notation & Value & Unit \\
\hline$X_{\mathrm{r}}$ & 1.8 & $\mathrm{~m}$ & $X_{0}$ & 0 & $\mathrm{~m}$ \\
$Y_{\mathrm{bbp}}$ & 3.2 & $\mathrm{~m}$ & $Y_{0}$ & 0.7 & $\mathrm{~m}$ \\
$X_{\text {ou }}$ & 23.5 & $\mathrm{~m}$ & $X_{t_{f}}$ & 100 & $\mathrm{~m}$ \\
$X_{\mathrm{od}}$ & 36.5 & $\mathrm{~m}$ & $Y_{t_{f}}$ & 1.4 & $\mathrm{~m}$ \\
& & & $v_{0}$ & 70 & $\mathrm{~km} / \mathrm{h}$ \\
\hline
\end{tabular}

where $X_{\mathrm{ou}}$ and $X_{\mathrm{od}}$ correspond to the points of the maximum increase and decrease, respectively, of the righthand side boundary along the $Y$-direction (see Figure 4), $X_{\mathrm{r}}$ defines the sharpness of the transition for both obstacle edges, and $Y_{\mathrm{bbp}}$ is the peak value of the boundary. Numerical values of the obstacle parameters are given in the left column in Table 2. Note that for these parameters the obstacle length is $11 \mathrm{~m}$, the same length as the obstacle length in Figure 4 and the distance between the obstacle cones in Figure 1. The bottom plot in Figure 4 shows the road and the vehicle representation used in the following formulation of the optimization problem. The obstacle boundaries are represented using (33) and the allowed lateral maneuvering region (i.e. along $Y$ ) for the vehicle center of mass is set to be between 0 and $1.4 \mathrm{~m}$ for the own driving lane, and correspondingly to be between 3.2 and $4.6 \mathrm{~m}$ for the opposing driving lane.

\section{Optimal Control Problem}

The motion-planning problem for the double lane-change maneuver is represented as an optimal control problem. The lane-deviation penalty (LDP) function ${ }^{13}$, associating a cost for driving in the opposing lane, and a recovery-behavior extension ${ }^{14}$, promoting smooth return to the original lane, are adopted in the criterion. The particular choice of the parameters for the road configuration is defined in this section, and the resulting maneuver for the nominal parameters is subsequently presented.

\section{Formulation}

The lane-deviation penalty (LDP) function ${ }^{13}$ is formulated using the auxiliary function (32) as

$$
H_{Y_{\mathrm{o}}}=\widetilde{H}_{Y_{\mathrm{o}}}^{Y_{\mathrm{r}}}(Y(t))
$$

where $Y_{\mathrm{o}}$ and $Y_{\mathrm{r}}$ are parameters. A projection of the LDP function on the $Y$-axis is illustrated with the thick curve in Figure 6a, where $Y_{\mathrm{o}}$ is set to the middle of the adjusted road width and $Y_{\mathrm{r}}$ is equal to the car width. This choice of parameters means that the penalty function $H_{Y_{\mathrm{o}}}$ gives a very low penalty when the vehicle is in its own lane and gives a high penalty in the opposing lane. A smooth switching

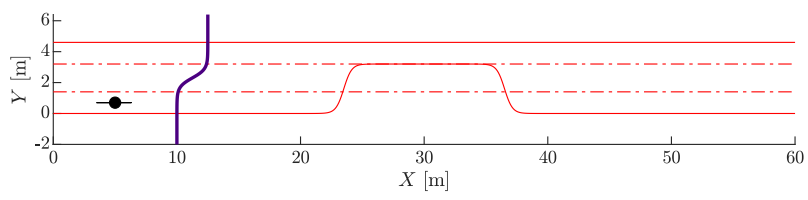

(a) Avoidance part. The thick line shows a projection of the LDP function (34).

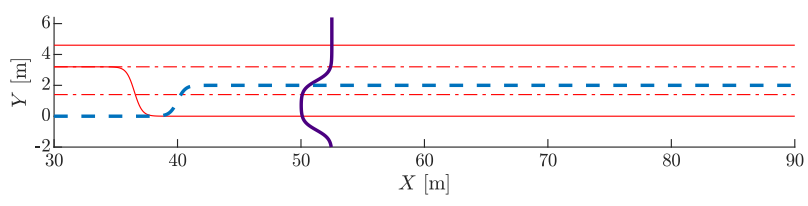

(b) Recovery part. The dashed thick line shows a projection of the switching function (35). The solid thick line shows a projection of (34) accompanied by the first term in the recovery-behavior extension (36).

Figure 6. Visualization of the maneuver setup. The obstacle and the right-hand side driving boundary (33) are shown for the nominal parameters in Table 2.

function at a point after the obstacle $\left(X_{1}=X_{\text {od }}+X_{\text {shift }}\right)$ is defined using (32) as

$$
H_{X_{1}}=\widetilde{H}_{X_{1}}^{X_{\mathrm{r}}}(X(t)) .
$$

A projection of $H_{X_{1}}$ on the $X$-axis is shown with the dashed thick line in Figure 6b.

In addition to the avoidance formulation (35) effective before the obstacle, a recovery-behavior extension ${ }^{14}$ is adopted. It aims for a smooth and swift motion in the recovery phase after the obstacle, and it includes five terms that have the following purposes: to complement the LDP function (34) such that the vehicle is promoted to drive in the middle of its own driving lane after the obstacle (see the solid thick line in Figure $6 \mathrm{~b}$ for a projection of this combination); to promote velocity recovery using a small penalty on the velocity deviation; to promote straight driving by including a small time penalty, which from many possible constant-velocity paths favors the straight path; two terms to include regularization of inputs to address actuatorredundancy singularities ${ }^{14}$. The sum of the terms multiplied with $H_{X_{1}}$, which makes these terms to be active only after the obstacle, defines the recovery-behavior extension ${ }^{14}$

$$
\begin{aligned}
R_{X_{1}}= & H_{X_{1}}\left(1-H_{Y_{1}}\right)+H_{X_{1}}\left(p_{v}\left(v-v_{\mathrm{ref}}\right)^{2}+\right. \\
& \left.\gamma+p_{T}\left(T_{1}^{2}+T_{2}^{2}+T_{3}^{2}+T_{4}^{2}\right)+p_{\delta} \delta^{2}\right)
\end{aligned}
$$

where $Y_{1}, p_{v}, \gamma, p_{T}$, and $p_{\delta}$ are parameters.

The maneuver to be performed is defined in the following way. The vehicle starts at the point $\left(X_{0}, Y_{0}\right)$ with the velocity $v_{0}$ and zero heading angle $(\psi=0)$. The final point is defined to be at $X_{t_{f}}$ and below $Y_{t_{f}}$, where the last condition is imposed, such that the vehicle ends at an appropriate lane position. Numerical values defining the maneuver are given in the right column in Table 2 . The final point $X_{t_{f}}$ is set to a distance that is further away than defined by the maneuver illustrated in Figure 1. The additional longitudinal distance is added to verify that the vehicle returns to its own driving lane and could follow it during the stabilization part of the maneuver.

The desired optimal trajectories are computed as the solution to an optimization problem. In this optimization 
problem, the objective to be minimized is an integral of the sum of (34) and (36) as in (37), and to complete the problem formulation, model constraints on vehicle, obstacle, and road from Section "Modeling" are recollected together with initial and terminal conditions in (38)-(45). The motion-planning problem for the studied double lane-change maneuver is consequently formulated as a continuous-time optimization problem in the following way:

$$
\begin{array}{cl}
\text { minimize } & \int_{0}^{t_{f}}\left(H_{Y_{\mathrm{o}}}+R_{X_{1}}\right) \mathrm{d} t \\
\text { subject to } & T_{i, \min } \leq T_{i} \leq H_{X_{1}} T_{i, \max } \\
& \left|\dot{T}_{i}\right| \leq \dot{T}_{i, \max }, i \in\{1,2,3,4\} \\
& |\delta| \leq \delta_{\max },|\dot{\delta}| \leq \dot{\delta}_{\max } \\
& X(0)=X_{0}, Y(0)=Y_{0}, \psi(0)=0 \\
& v_{X}(0)=v_{0}, v_{Y}(0)=0 \\
& X\left(t_{f}\right)=X_{t_{f}}, Y\left(t_{f}\right) \leq Y_{t_{f}} \\
& Y_{\mathrm{bb}}(X(t)) \leq Y(t), \\
& \dot{\mathbf{x}}=G(\mathbf{x}, \mathbf{z}, \mathbf{u}), h(\mathbf{x}, \mathbf{z}, \mathbf{u})=0
\end{array}
$$

where $t_{f}$ is the total time of the maneuver, which is unknown a priori and thus part of the optimization problem. The limit values for the torques and steering are set as described in Section "Modeling". The upper limit in (38) contains $H_{X_{1}}$, which makes the maximum torque to be zero in the avoidance part and to be according to the model limits (13)-(14) in the recovery part. The right-hand side driving boundary (33) is imposed as a constraint. The left-hand side driving boundary is not included in the optimization formulation since the LDP function itself acts as a soft constraint in that respect and an explicit constraint is thus not needed for the used objective function. The vehicle model (30)-(31) also needs to be fulfilled.

Many parameters have been given values in Section "Modeling", and the remaining numerical values of the parameters are selected as in the previous research ${ }^{14}: Y_{\mathrm{o}}=$ $2.3 \mathrm{~m}, Y_{\mathrm{r}}=1.8 \mathrm{~m}, Y_{1}=-0.9 \mathrm{~m}, p_{v}=0.2, p_{T}=2 \cdot 10^{-11}$, $p_{\delta}=0.25, \gamma=0.25$, and $X_{\text {shift }}=3.5 \mathrm{~m}$.

\section{Implementation}

Numerical solutions to the optimization problem (37)-(45) were computed using the platform JModelica.org ${ }^{30}$ and the IPOPT software package for nonlinear optimization ${ }^{31}$, together with the MA57 linear solver ${ }^{32}$. Direct simultaneous collocation ${ }^{33}$ was used for transforming the continuous-time optimization problem (37)-(45) to a non-linear program to be solved by IPOPT. Further details on the implementation and optimization of the models are available in the article on the used optimization framework ${ }^{12}$.

\section{Initialization of Numerical Optimization}

Considering that the optimization problem to solve is nonconvex and that numerical iterative methods are used to search for a minimum, an initialization of the trajectories for the model variables is beneficial for the convergence of the numerical optimization. To solve the optimization problem (37)-(45) with the default parameters (Table 2), the optimization solver in JModelica.org is therefore provided
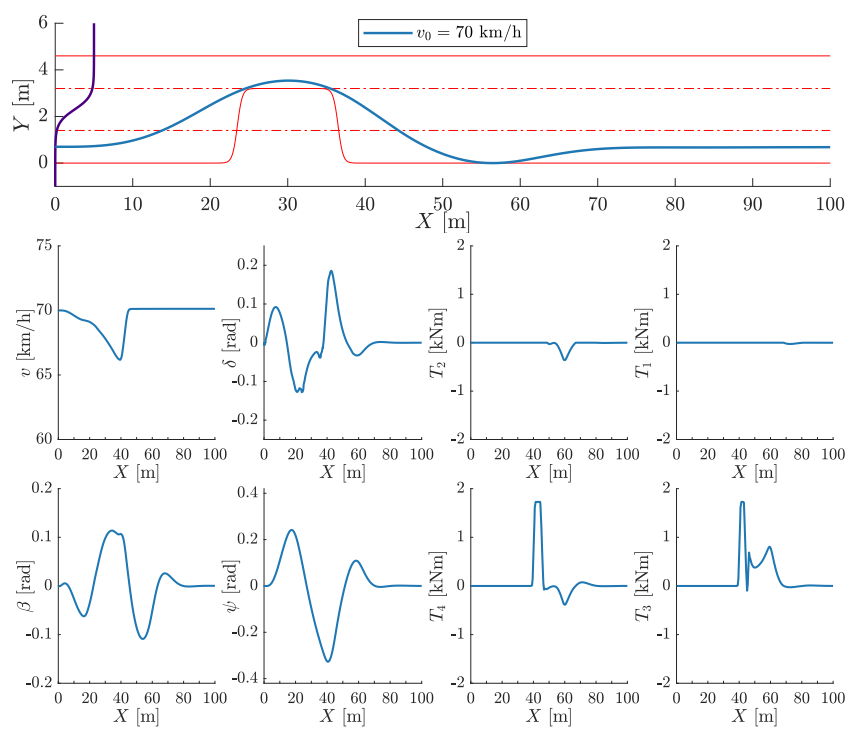

Figure 7. Paths and selected vehicle variables found for the nominal parameters (Table 2).

with an initial trajectory obtained by simulation of the same model as used in the optimization. The simulation is performed with such constant inputs that the vehicle avoids the obstacle by driving on a circular arc. In the simulation, the vehicle is set to the same initial velocity as in the optimization; the inputs (27) are zero, the initial value for each wheel torque is set to zero, so the vehicle is driving with nearly the same velocity for the complete maneuver. The initial vehicle orientation is set to

$$
\psi(0)=-\left(\arccos \left(\frac{X_{t_{f}}}{2 R}\right)-\frac{\pi}{2}\right),
$$

where $R$ is the radius of the chosen arc, which is a tuning parameter to obtain a desired initial trajectory. The used value of the radius is $R=300 \mathrm{~m}$. The initial steering angle is chosen as

$$
\delta(0)=-\frac{l_{f}+l_{r}}{R} .
$$

The simulation time for this initialization is set to

$$
t_{f}=\frac{X_{t_{f}}}{v_{0}} .
$$

The numerical integration of the vehicle dynamics required to compute the simulated trajectories for initialization is obtained by using the simulation tools in the JModelica.org platform. It is the same platform as used for the optimization, and it takes care of the correct interpolation of the simulation results for use with the specified collocation configuration in the subsequent solution of the optimization problem. Solutions to the optimization problem (37)-(45) with parameter variations in the scenario were obtained by using the solution of the default formulation as initialization.

\section{Maneuver for Nominal Parameters}

Figure 7 shows the results obtained using the formulation (37)-(45) for the nominal parameters of the considered scenario (Table 2). The figure shows the path of the vehicle in the XY-plane, the vehicle velocity $v$, the vehicle orientation 
$\psi$, the body-slip angle $\beta$ obtained as

$$
\beta=\arctan \left(\frac{v_{y}}{v_{x}}\right),
$$

and the control inputs: steering $\delta$ and torques $T_{1}, T_{2}, T_{3}$, and $T_{4}$. In Figure 7 and in the following figures, the selected variables are shown as functions of $X$ to make the study of the vehicle behavior relative to the obstacle position easier, as well as to allow comparisons of different formulations. The $Y$-axis is scaled up relative to the $X$-axis in the top plot of Figure 7 and in the following figures to emphasize differences in the lateral behavior of the vehicle. As specified in the formulation (37)-(45), the torque is non-positive in the avoidance phase ( $X$ before $X_{1}=40 \mathrm{~m}$ ). For this setup, the optimal solution is to not apply braking in the avoidance phase to minimize the time in the opposing lane. The velocity decrease owing to the vehicle dynamics is mitigated by applying driving torque in the recovery phase. Overall, the vehicle is fast to return to the original lane after having avoided the obstacle and thus minimizing the risk of a potential collision with an oncoming vehicle.

\section{Scenario Variations}

It is of interest to study the resulting trajectories for different scenarios regarding obstacle and vehicle. In this section, variations of the problem are investigated for the obstacle width and length, for the distance to the obstacle, as well as for the initial velocity of the vehicle.

\section{Obstacle Width}

Variations in the obstacle width $w$, that is, the road width occupied by the obstacle along the $Y$-direction, are studied. The obtained paths and selected trajectories for the model variables are shown in Figure 8a. The same LDP objective (37) is used for all optimizations, with parameters $Y_{\mathrm{o}}$ and $Y_{1}$ that are motivated by the road and the vehicle width, but to account for the obstacle geometry, the bottom drivable area is modified, namely $Y_{\mathrm{bbp}}$ in (33). The characteristics of the $X Y$-paths are very similar when comparing different widths of the obstacle. An increase in the obstacle width results in a slight velocity decrease and in an expected increase in the absolute value of the maximum and the minimum values of the vehicle orientation $\psi$. The state variables for maneuvers with the width from 2.6 to $4 \mathrm{~m}$ have similar behavior. However, for the smallest obstacle width, $w=2.2 \mathrm{~m}$, the obtained maneuver is different from the other maneuvers. The difference is, for example, visible for the steering angle and vehicle orientation. Such behavior is the result of a steep gradient of the penalty function when passing close to a narrow obstacle (compare the slope of the penalty function at 3.2 and 2.2 in Figure 5). This makes steering towards the obstacle during the avoidance phase of the maneuver to be optimal, also affecting the vehicle lateral position, which is almost constant while passing the obstacle and not arc-like as for the other maneuvers.

\section{Obstacle Length}

The paths and the associated trajectories for selected model variables, obtained by variation of the length $l$ of the obstacle (i.e. the size of the obstacle along the $X$-direction) between 5 and $18 \mathrm{~m}$ are shown in Figure $8 \mathrm{~b}$. The length of the obstacle is the difference between $X_{\mathrm{od}}$ and $X_{\mathrm{ou}}$ in (33) adjusted for $X_{\mathrm{r}}$ (see Figure 4) as

$$
l=X_{\mathrm{od}}-X_{\mathrm{ou}}-X_{\mathrm{r}} .
$$

Different lengths of the obstacle are achieved by updating $X_{\text {od }}$. For the considered optimization formulation, it is beneficial to immediately return to the original lane once the obstacle is passed. In all obtained maneuvers, the shape of the $X Y$-path is such, that the path touches the farthermost corner of the obstacle. Information about the obstacle length in the optimizations, results in different optimal steering strategies and different velocity profiles. Longer obstacles naturally result in a later point for the rapid steering-direction change (see the range $X=30-50 \mathrm{~m}$ for the steering angle $\delta$ in Figure 8b). The minimum for the velocity shifts to the right with the increase of the obstacle length as well, since the switching point $X_{1}$ is set to be the fixed distance $X_{\text {shift }}$ after the obstacle end $X_{\text {od }}$.

\section{Initial Velocity}

Maneuvers were computed with different initial velocities $v_{0}$, and the results are shown in Figure $8 \mathrm{c}$. The maneuvers obtained for the initial velocities from $v_{0}=50$ to $80 \mathrm{~km} / \mathrm{h}$ show good performance both in the avoidance and the recovery parts. The maneuver with the initial velocity $v_{0}=$ $90 \mathrm{~km} / \mathrm{h}$ is possible to perform, but a longer distance is required after the obstacle for the vehicle to finish the recovery phase, and a slower return to the original lane compared to the maneuvers with lower initial velocities is observed. The lower initial velocity, the smaller turning radius is possible given the available lateral force, which in most cases results in a higher maximum lateral displacement (compare $Y$ for $v_{0}=50$ and $70 \mathrm{~km} / \mathrm{h}$ at $X=30 \mathrm{~m}$ ). This behavior is optimal since, even though there is a slightly higher lateral displacement, the considered optimization criterion prioritizes a steeper return to the own lane leading to a reduced time in the opposing lane.

\section{Distance to the Obstacle}

Paths and selected variables for maneuvers with different initial distances $d$ to the obstacle are presented in Figure 8d. The distance to the obstacle is defined as

$$
d=X_{\text {ou }}+\frac{X_{\mathrm{r}}}{2} .
$$

The length of the obstacle is kept to the nominal value for all considered cases in Figure 8d, and different distances are obtained by adjusting both $X_{\mathrm{ou}}$ and $X_{\mathrm{od}}$. The major parts of the state-variable trajectories for maneuvers with distances 22-40 m are very similar to each other, with some shift along the $X$-axis. However, for the maneuver with $d=20 \mathrm{~m}$, the vehicle changes its behavior relative to the other obtained maneuvers by applying more braking and steering before the obstacle because of the shorter available distance to the obstacle.

\section{Discussion: Overall Motion Planning}

The results for the different scenario variations shown in Figure 8 have some common structures for the obtained 

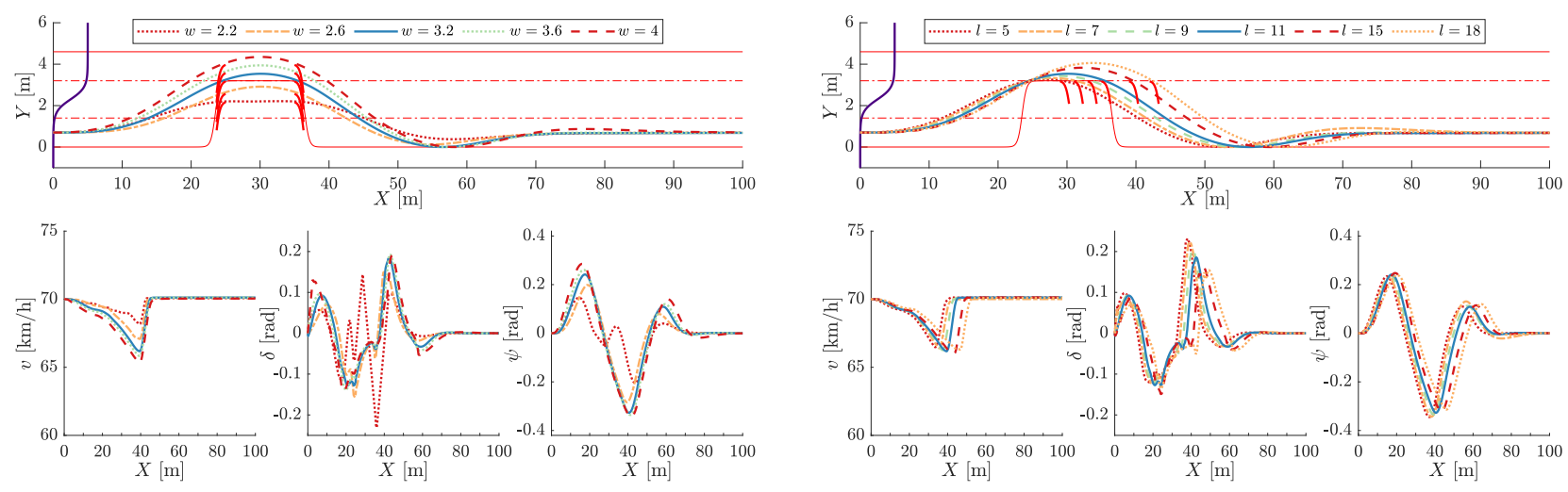

(a) Obstacle widths $w$ (in meters).

(b) Lengths $l$ of the obstacle (in meters).
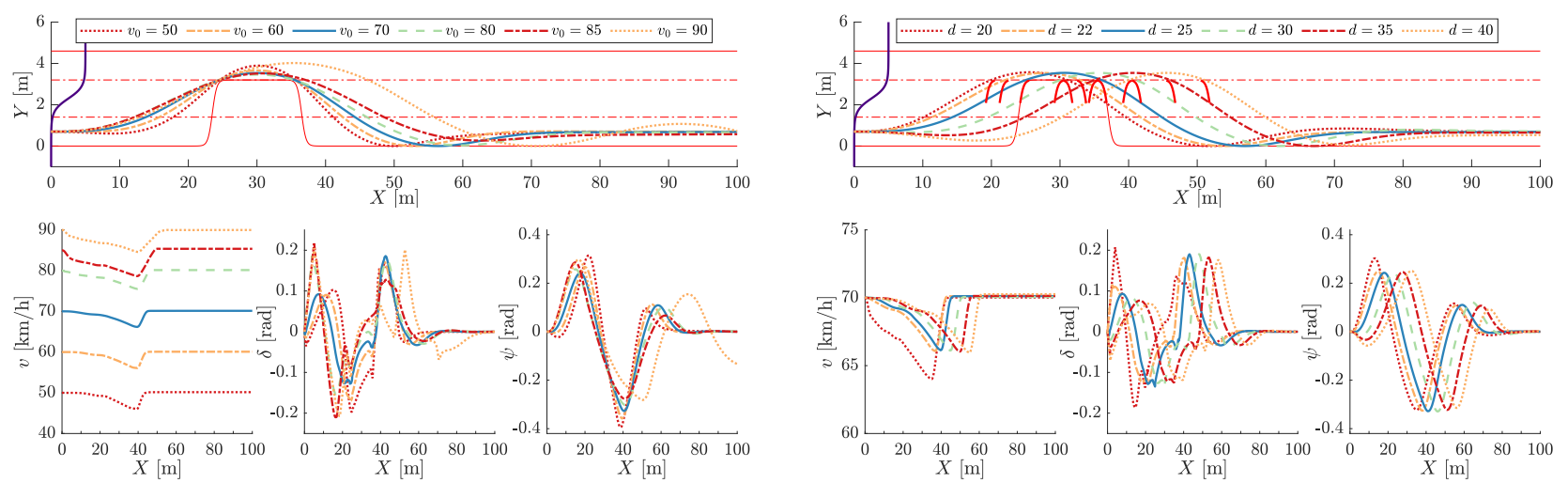

(c) Initial velocities $v_{0}(\mathrm{~km} / \mathrm{h})$.

(d) Obstacle distances $d$ (in meters).

Figure 8. Paths and selected vehicle variables found for different scenario variations. The nominal parameters are according to Table 2. The thick arcs show the boundaries of the obstacle for the respective modified parameters.

LDP maneuvers that can be put into a general perspective regarding the potential of LDP maneuvers in a maneuverbased motion planner. Such a motion planner may choose between different actions and select parameters for the chosen action. Remarks on such aspects are the topic of the next three subsections. Further remarks are presented in Section "Discussion".

Structure in the Solutions The LDP maneuvers obtained for different scenario variations show that the paths and the state variables exhibit structured changes for the different optimizations with the LDP objective (see, e.g. Figures $8 \mathrm{~b}$ and 8d, where one solution can almost be obtained from another by appropriate scaling and shifting of the resulting state variables in most cases). The observed similarity property is beneficial for developing a maneuver-based online motion planner, where maneuvers for different driving conditions and different initial values can be obtained by interpolation of offline pre-computed optimization solutions. Hence, the LDP formulation (37) can be used to equip a maneuver-based planner, where the obtained maneuvers transfer the vehicle from normal operation via avoidance and recovery phases, back to normal operation. Since the computed maneuvers exhibit structured changes for variations of the parameters of the scenario, only a rather small number of certain characteristic maneuvers are needed to be pre-computed, saving computation time and also decreasing the required storage, in a maneuver-based motion planner.

Emergency Braking In the case of a sudden appearance of an obstacle in the way of a vehicle, there could be different strategies for a motion planner to try to handle the situation, and one strategy would be to start with an emergency braking before making an avoidance maneuver. For the velocities $v_{0}=50$ to $v_{0}=90 \mathrm{~km} / \mathrm{h}$, the braking distance varies from 9.0 to $28.1 \mathrm{~m}$. For the nominal configuration (Table 2), the distance to the obstacle is $24.4 \mathrm{~m}$. For low speeds it would thus be possible for the vehicle to stop before the obstacle, but for the higher velocities the vehicle is not able to stop before the obstacle. For these cases, the LDP formulation (as many other obstacle-avoidance formulations) offers a solution to successfully perform an avoidance maneuver. Consequently, the LDP can handle more cases than emergency braking. However, from the perspective of the motion planner the main point is that, if viewing $v_{0}$ as the remaining velocity after initial braking, both approaches can advantageously be combined.

Pendulum-Like Motion With the increase of the available distance to the obstacle, it gets more pronounced that the vehicle exhibits pendulum-like motion where the vehicle first drives to the right relative to its direction of motion and then drives to the left to avoid the obstacle. This is a technique used by race drivers, before a maneuver, to build up the rotation of the vehicle to be able to perform the 
maneuver faster. The effect is visible for the steering angle and the vehicle orientation being negative at the beginning of the maneuver (i.e. the vehicle orientation $\psi$ and the steering angle $\delta$ of the maneuver decrease as observed in Figure $8 d$ ). Such a behavior is also observed for the lower initial velocities $(50-60 \mathrm{~km} / \mathrm{h})$ in Figure $8 \mathrm{c}$.

The scale of the pendulum-like motion is here limited by the width of the initial straight road segment considered in this double lane-change scenario, and by the non-positive torque constraint during the avoidance part of the maneuver. These constraints make the scale of the observed behavior to be smaller than for pendulum-turn maneuvers from race driving experiments ${ }^{34}$. More flexibility in the use of the actuators, without the non-positive torque constraint, was considered in a previous study ${ }^{13}$, and it was observed that the pendulum-like motion then is more pronounced. Further, it was there computed, that when the pendulum action is prohibited by a steering constraint, the time in the opposing lane increases by $2.5 \%$ for the initial velocity $v_{0}=50 \mathrm{~km} / \mathrm{h}$. In the study here, with the same steering constraint and with the reduced flexibility in use of the actuators in the avoidance phase (the non-positive torque constraint in the formulation (37)-(45)), this increase is $0.8 \%$ for the initial velocity $v_{0}=50 \mathrm{~km} / \mathrm{h}$. In conclusion, pendulum action may because of its swiftness increase safety by reducing the time in the opposing lane, but there may also be risks with a more aggressive maneuver. Thus, depending on the situation, the motion planner could allow more or less pendulum action.

\section{Comparison with Other Criteria}

Solutions to the considered problem using the LDP objective function are compared with solutions obtained using other formulations: a squared lateral-error norm, the Huber loss criterion, and a minimum-time objective function. The alternative formulations are presented with the recoverybehavior extension ${ }^{14}$ for improved performance in the recovery phase. Then, the obtained maneuvers for different formulations are compared with each other in terms of time spent in the opposing lane, maximum acceleration for the maneuver, and behavior differences.

\section{Minimum-Time Formulation}

The objective function for the minimum-time optimization is here formulated as

$$
\int_{0}^{t_{f}}\left(p_{t}+\breve{R}_{X_{1}}\right) \mathrm{d} t
$$

where $p_{t}$ is a time-penalty parameter. Compared to (36), the recovery-behavior extension term $\breve{R}_{X_{1}}$ is here formulated as

$$
\breve{R}_{X_{1}}=H_{X_{1}}\left(p_{T}\left(T_{1}^{2}+T_{2}^{2}+T_{3}^{2}+T_{4}^{2}\right)+p_{\delta} \delta^{2}\right)
$$

where the velocity deviation penalty is substituted with a velocity constraint

$$
v \leq v_{\text {ref }}
$$

The term $H_{X_{1}} p_{v}\left(v-v_{\text {ref }}\right)^{2}$ is not needed in this formulation since the time-penalty term $p_{t}$ penalizes velocity decrease below the maximum allowed velocity $v_{\text {ref }}$. On the other hand, the penalties on the wheel torques and on the steering angle are applied as in (36) ${ }^{14}$.

When the same driving boundary (44) as for the LDP case is used, the minimum-time formulation (52)-(54) results in paths where the vehicle is comparably slower to return to the original lane. To make the formulations more comparable, a minimum-time with a top boundary formulation is considered instead, where an additional lanechange boundary is added that is formulated as

$Y(t) \leq Y_{\mathrm{tb}}$,

$Y_{\mathrm{tb}}(X(t))=Y_{\mathrm{lw}}+Y_{\mathrm{tbp}}\left(\widetilde{H}_{X_{\mathrm{otu}}}^{X_{\mathrm{r}}}(X(t))-\widetilde{H}_{X_{\mathrm{otd}}}^{X_{\mathrm{r}}}(X(t))\right)$,

where $Y_{\mathrm{lw}}$ is the adjusted lane width and $X_{\text {otu }}, X_{\text {otd }}$ are the points where the top boundary goes up and down, respectively. The values of these parameters used in the optimization are $1.4 \mathrm{~m}, 12 \mathrm{~m}$, and $47 \mathrm{~m}$, respectively.

\section{Squared Lateral-Error Norm Formulation}

An alternative approach to formulate the objective function is evaluated by penalizing an integral squared-error norm of lateral displacement that was used in the previous research ${ }^{11}$ for the recovery part of the double lane-change maneuver. Here, such a penalty function is applied for the full maneuver by using the following objective function

$$
\int_{0}^{t_{f}}\left(p_{s}\left(Y_{0}-Y\right)^{2}+\bar{R}_{X_{1}}\right) \mathrm{d} t
$$

where $p_{s}$ is a weighting factor and

$$
\begin{aligned}
\bar{R}_{X_{1}}=H_{X_{1}} & \left(p_{v}\left(v-v_{\text {ref }}\right)^{2}+\gamma\right. \\
& \left.+p_{T}\left(T_{1}^{2}+T_{2}^{2}+T_{3}^{2}+T_{4}^{2}\right)+p_{\delta} \delta^{2}\right) .
\end{aligned}
$$

Compared to (37), the LDP penalty $H_{Y_{0}}$ is replaced by the lateral displacement penalty $\left(Y_{0}-Y\right)^{2}$. This penalty has a minimum at $Y_{0}$ and therefore favors driving in the middle of the lane, so an extra term $H_{X_{1}}\left(1-H_{Y_{1}}\right)$ after the obstacle is not needed here (compare (58) with (36)). However, other terms of the behavior extension, for example, $H_{X_{1}} p_{v}(v-$ $\left.v_{\text {ref }}\right)^{2}$, are still needed to obtain the desired vehicle behavior after the obstacle in the velocity-recovery phase and to obtain satisfactory torque distribution between the front and the rear wheels ${ }^{14}$.

\section{Huber Formulation}

It is further considered formulating the objective function as an integral of the Huber cost function ${ }^{35}$ applied to the lateral displacement. The Huber cost function is a hybrid between absolute error and squared error. To allow continuous derivatives of all orders, a very similar cost function to the Huber cost function is used, which is called the Pseudo-Huber cost function ${ }^{36}$

$$
C_{b}(\delta)=2 b^{2}\left(\sqrt{1+\left(\frac{\delta}{b}\right)^{2}}-1\right)
$$

where $2 b$ defines the linear slope of the penalty for large $\delta$. The Huber Formulation is here defined with the following 


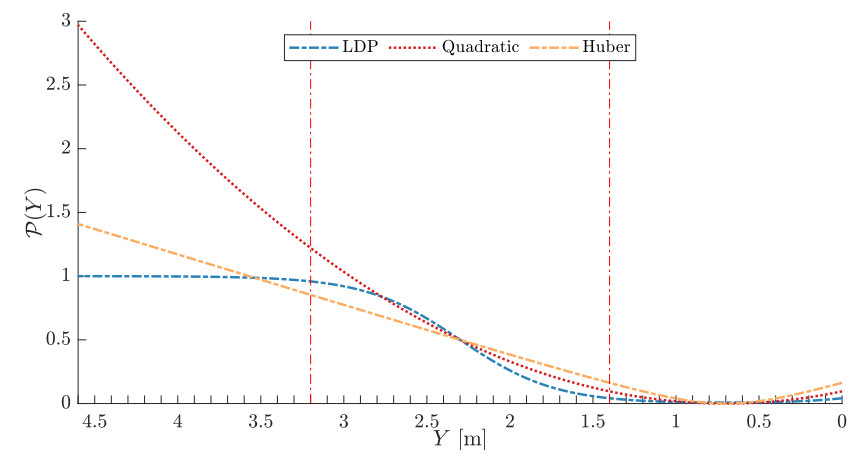

Figure 9. Illustration of the used lateral penalties.

objective function

$$
\int_{0}^{t_{f}}\left(C_{b}(Y)+\bar{R}_{X_{1}}\right) \mathrm{d} t
$$

where the recovery-behavior extension $\bar{R}_{X_{1}}$ is defined in (58).

\section{Weighting Factors}

The weighting factors $p_{t}$ and $p_{s}$, and the tuning parameter $b$ in the Pseudo-Huber cost function are chosen to balance the relative influence of the affected terms and the behaviorextended part. The factor $p_{t}$ is $1 / 9$. The values $p_{s}$ and $b$ are set to such values that the penalties have the cost equal to 0.5 at $Y=2.3 \mathrm{~m}$ (see Figure 9), which for the considered case means that $p_{s}=0.2$ and $b=0.4$. The lateral position $Y=2.3 \mathrm{~m}$ corresponds to the situation when the vehicle is in-between two lanes and the associated cost for that position is the same as for the LDP formulation.

\section{Comparison}

Figure 10 compares maneuvers for the different formulations, found for $v_{0}=50$ and $70 \mathrm{~km} / \mathrm{h}$. All lateral penaltybased formulations exhibit similar characteristics in terms of avoidance behavior. At high initial velocities, the available actuation is mostly utilized to fulfill the optimization constraints (to avoid the obstacle), which leaves limited room to optimize a chosen criterion. The result is almost identical paths in the avoidance phase for all lateral penalty-based formulations at $v_{0}=70 \mathrm{~km} / \mathrm{h}$. The recovery-behavior terms give good behavior in the recovery phase in all considered formulations. A more aggressive steering after the obstacle is obtained with the squared-error norm and Huber formulations, since the vehicle tries to return to the original lateral position, not just to its own lane, which also results in a higher amplitude of the yaw rate $\dot{\psi}$ (not shown in the figures).

Table 3 compares the time that the vehicle is outside of its own lane for the different optimization criteria for initial velocities from $v_{0}=50$ to $80 \mathrm{~km} / \mathrm{h}$. Optimizations with the LDP formulation give paths and trajectories that are safer in terms of the time that the vehicle stays outside its own lane. For $v_{0}=50 \mathrm{~km} / \mathrm{h}$, the minimum-time, squared-error norm, and Huber formulations result in $48.6 \%, 13.5 \%$, and $4.3 \%$ higher time in the opposing lane, respectively, compared to the LDP formulation.

The lateral accelerations $a_{y}$ in Figure 10 are very similar for the lateral penalty-based formulations, with differences
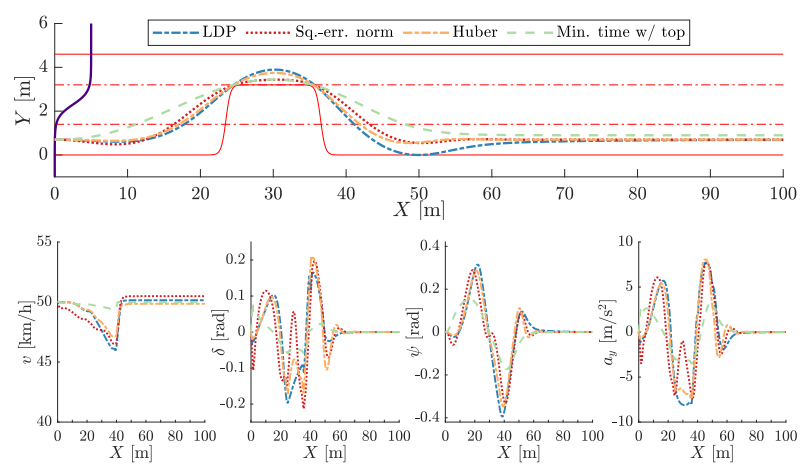

(a) Initial velocity $v_{0}=50 \mathrm{~km} / \mathrm{h}$.
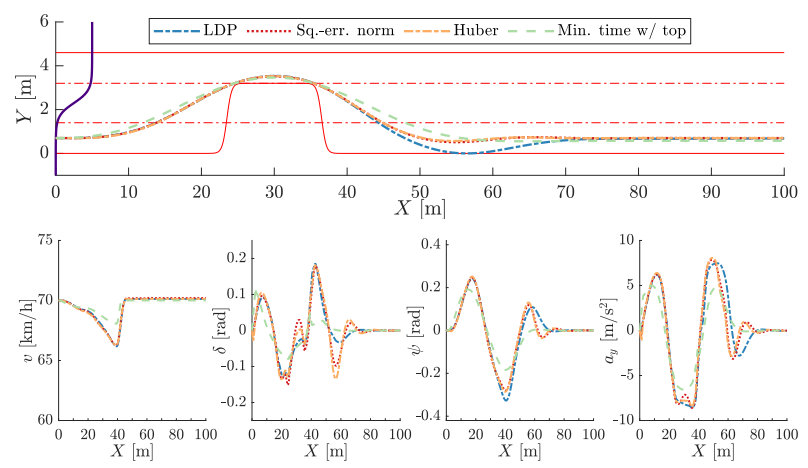

(b) Initial velocity $v_{0}=70 \mathrm{~km} / \mathrm{h}$.

Figure 10. Comparison of maneuvers for different objectives.

Table 3. Time (in s) that the vehicle is outside of its own lane $\left(Y>Y_{0}\right)$ for different initial velocities and problem formulations in the optimization.

\begin{tabular}{c|cccc}
\hline Name $\backslash v_{0}$ & $50 \mathrm{~km} / \mathrm{h}$ & $60 \mathrm{~km} / \mathrm{h}$ & $70 \mathrm{~km} / \mathrm{h}$ & $80 \mathrm{~km} / \mathrm{h}$ \\
\hline LDP & 1.81 & 1.69 & 1.63 & 1.59 \\
Min. time & 2.69 & 2.23 & 1.89 & 1.66 \\
Sq.-err. norm & 2.05 & 1.75 & 1.66 & 1.62 \\
Huber & 1.89 & 1.73 & 1.66 & 1.62 \\
\hline
\end{tabular}

for the area $X=25-40 \mathrm{~m}$, where the vehicle steers more aggressively ( $\delta$ in Figure 10 ) by trying to minimize the lateral distance to the obstacle in the results obtained with the squared-error norm and the Huber formulations. The minimum-time formulation in general results in maneuvers with lower amplitude of the lateral acceleration $a_{y}$ than the other considered formulations. The same observation of difference in aggressiveness is valid for the maximum value of the Euclidean norm of the acceleration vector,

$$
\sqrt{a_{x}^{2}+a_{y}^{2}}
$$

shown in Table 4. This difference, however, is smaller at higher initial velocities (compare the values of the lateral acceleration for LDP and the minimum-time with top boundary in Table 4 for increasing initial velocities). For low initial velocities, when actuation has higher flexibility since it is less restricted by the obstacle avoidance, the LDP formulation provides maneuvers with shorter time outside the own lane of the vehicle. 
Table 4. Maximum value of the Euclidean norm of the acceleration vector (in $\mathrm{m} / \mathrm{s}^{2}$ ) for different initial velocities and problem formulations in the optimization.

\begin{tabular}{c|cccc}
\hline Name $\backslash v_{0}$ & $50 \mathrm{~km} / \mathrm{h}$ & $60 \mathrm{~km} / \mathrm{h}$ & $70 \mathrm{~km} / \mathrm{h}$ & $80 \mathrm{~km} / \mathrm{h}$ \\
\hline LDP & 8.13 & 8.44 & 8.61 & 8.57 \\
Min. time & 3.52 & 4.82 & 6.59 & 8.09 \\
Sq.-err. norm & 7.72 & 7.96 & 8.61 & 8.69 \\
Huber & 8.08 & 8.19 & 8.46 & 8.44 \\
\hline
\end{tabular}

\section{Discussion}

The primary objective of this paper is to develop and analyze the lane-deviation penalty for computing double lane-change maneuvers, giving a foundation for improved control of vehicle safety maneuvers in at-the-limit situations. Several new insights were presented, and the resulting convincing behavior observed in the maneuvers naturally raises the questions of implementation and real-time control based on the results. Several directions for achieving this could be considered. As described in Section "Structure in the Solutions", using a library of offline pre-computed parameterized double lane-change maneuvers based on the developed LDP objective in a maneuver-based motion planner would be a possible application of the results. In light of the increased computational capacity onboard an autonomous vehicle and improvements in computational times for optimization algorithms, real-time computation of the maneuvers could be within reach in the near future, especially if segmentation techniques, such as the one from Anistratov et al. ${ }^{15}$, are applied to reduce the computational time further. Another appealing approach is to apply receding-horizon control with LDP as objective in the optimization problem solved over the finite prediction horizon. Adopting a strategy such as in Fors ${ }^{37}$ with a twolevel architecture can then enable real-time control. There, an optimization problem with a low-complexity model is solved at the higher level, resulting in an acceleration profile that is subsequently refined in several steps considering more detailed vehicle dynamics to result in the actual actuator inputs in the lower level. The developed LDP objective can there be used in the higher level when computing the acceleration profile to define the desired behavior in the double lane-change maneuver.

\section{Conclusions}

The lane-deviation penalty (LDP) formulation, penalizing being in the opposing driving lane, was developed for computing optimal double lane-change maneuvers. The developed framework is utilizing optimal all-wheel braking and steering at the limit of road-tire friction, and it is analyzed using a double-track vehicle model with load transfer. The resulting behavior was given a careful and thorough treatment in several interesting cases, where parameters of the emergency situation such as the initial speed of the vehicle and the size and placement of the obstacle are varied. The overall conclusion is that the formulation performs well for velocities ranging from the low end, where it may be possible to do emergency braking, to the high end, where the speed is on the border of being too high to handle the obstacle satisfactorily. The result is efficient and stable maneuvers. Another interesting contribution is the comparison with other criteria being a minimum-time formulation and two other lateral-penalty formulations. By design the LDP function is parameterized using information about the road and car widths, and, even though the LDP is a penalty on position, it results in maneuvers with less time in the opposing lane than the other criteria. This is positive since it leads to the desired effect of decreasing the danger of a potential collision with opposing traffic.

\section{Funding}

This work was partially supported by the Wallenberg AI, Autonomous Systems and Software Program (WASP) funded by the Knut and Alice Wallenberg Foundation.

\section{References}

1. SAE J3016. Taxonomy and definitions for terms related to driving automation systems for on-road motor vehicles. Technical report, SAE International. USA, 2018.

2. National Transportation Safety Board. NTSB news release: Tesla crash investigation yields 9 NTSB safety recommendations. https://www.ntsb.gov/news/ press-releases/Pages/NR20200225.aspx, 2020. (Date accessed: 16.09.2020).

3. Subosits $\mathbf{J}$ and Gerdes JC. From the racetrack to the road: Real-time trajectory replanning for autonomous driving. IEEE Transactions on Intelligent Vehicles 2019; 4(2): 309-320.

4. Brown M and Gerdes JC. Coordinating tire forces to avoid obstacles using nonlinear model predictive control. IEEE Transactions on Intelligent Vehicles 2020; 5(1): 21-31.

5. Svensson L, Bujarbaruah M, Kapania NR et al. Adaptive trajectory planning and optimization at limits of handling. In IEEE/RSJ International Conference on Intelligent Robots and Systems (IROS). Macau, China, pp. 3942-3948. 2019.

6. Fors V, Olofsson B and Nielsen L. Attainable force volumes of optimal autonomous at-the-limit vehicle manoeuvres. Vehicle System Dynamics 2020; 58(7): 1101-1122.

7. Olofsson B and Nielsen L. Using crash databases to predict effectiveness of new autonomous vehicle maneuvers for lanedeparture injury reduction. IEEE Transactions on Intelligent Transportation Systems 2020; : 1-12URL https://doi. org/10.1109/tits.2020.2983553.

8. ISO 3888-2:2011. Passenger cars - Test track for a severe lane-change manoeuvre - Part 2: Obstacle avoidance. Technical report, International Organization for Standardization (ISO). Geneva, Switzerland, 2011.

9. Shiller Z and Sundar S. Emergency lane-change maneuvers of autonomous vehicles. Journal of Dynamic Systems, Measurement, and Control 1998; 120(1): 37-44.

10. Gerdes JC and Rossetter EJ. A unified approach to driver assistance systems based on artificial potential fields. Journal of Dynamic Systems, Measurement, and Control 2001; 123(3): 431-438.

11. Dingle $P$ and Guzzella L. Optimal emergency maneuvers on highways for passenger vehicles with two- and four-wheel active steering. In American Control Conference. Baltimore, MD, USA, pp. 5374-5381. 2010.

12. Berntorp K, Olofsson $\mathrm{B}$, Lundahl $\mathrm{K}$ et al. Models and methodology for optimal trajectory generation in safety-critical 
road-vehicle manoeuvres. Vehicle System Dynamics 2014; 52(10): 1304-1332.

13. Anistratov P, Olofsson B and Nielsen L. Lane departure-based penalty for autonomous avoidance maneuvers. In Proceedings of the 14th International Symposium on Advanced Vehicle Control (AVEC). Beijing, China. 2018.

14. Anistratov P. Computation of autonomous safety maneuvers using segmentation and optimization. Linköping studies in science and technology. Licentiate Thesis 1860, Linköping University, Department of Electrical Engineering, 2019.

15. Anistratov P, Olofsson B and Nielsen L. Segmentation and merging of autonomous at-the-limit maneuvers for ground vehicles. In Proceedings of the 14th International Symposium on Advanced Vehicle Control (AVEC). Beijing, China. 2018.

16. Gao Y, Gordon T and Lidberg M. Optimal control of brakes and steering for autonomous collision avoidance using modified Hamiltonian algorithm. Vehicle System Dynamics 2019; 57(8): 1224-1240.

17. Gao $\mathrm{Y}$ and Gordon T. Optimal control of vehicle dynamics for the prevention of road departure on curved roads. IEEE Transactions on Vehicular Technology 2019; 68(10): 93709384.

18. Arikere A, Yang D, Klomp $M$ et al. Integrated evasive manoeuvre assist for collision mitigation with oncoming vehicles. Vehicle System Dynamics 2018; 56(10): 1577-1603.

19. Liu K, Gong J, Kurt A et al. Dynamic modeling and control of high-speed automated vehicles for lane change maneuver. IEEE Transactions on Intelligent Vehicles 2018; 3(3): 329-339.

20. Funke J, Brown M, Erlien SM et al. Collision avoidance and stabilization for autonomous vehicles in emergency scenarios. IEEE Transactions on Control Systems Technology 2017; 25(4): 1204-1216.

21. Zhang J, Sun W and Du H. Integrated motion control scheme for four-wheel-independent vehicles considering critical conditions. IEEE Transactions on Vehicular Technology 2019; 68(8): 7488-7497.

22. Rossetter E, Switkes J and Gerdes J. Experimental validation of the potential field lanekeeping system. International Journal of Automotive Technology 2004; 5(2): 95-108.

23. Ji J, Khajepour A, Melek WW et al. Path planning and tracking for vehicle collision avoidance based on model predictive control with multiconstraints. IEEE Transactions on Vehicular Technology 2017; 66(2): 952-964.
24. Berntorp K, Quirynen R, Uno T et al. Trajectory tracking for autonomous vehicles on varying road surfaces by frictionadaptive nonlinear model predictive control. Vehicle System Dynamics 2019; 58(5): 705-725.

25. Zeping $\mathrm{F}$ and Jianmin D. Optimal lane change motion of intelligent vehicles based on extended adaptive pseudo-spectral method under uncertain vehicle mass. Advances in Mechanical Engineering 2017; 9(7): 1-15.

26. Fors V, Olofsson B and Nielsen L. Formulation and interpretation of optimal braking and steering patterns towards autonomous safety-critical manoeuvres. Vehicle System Dynamics 2019; 57(8): 1206-1223.

27. Wong JY. Theory of Ground Vehicles. Fourth ed. Hoboken, NJ, USA: Wiley, 2008.

28. Pacejka H. Tyre and vehicle dynamics. Second ed. Oxford, UK: Butterworth-Heinemann, 2006.

29. Modelica Association. http://www.modelica.org, 2020. (Date accessed: 16.09.2020).

30. Åkesson J, Årzén KE, Gäfvert M et al. Modeling and optimization with Optimica and JModelica.org-Languages and tools for solving large-scale dynamic optimization problems. Computers and Chemical Engineering 2010; 34(11): 1737-1749.

31. Wächter A and Biegler LT. On the implementation of an interior-point filter line-search algorithm for large-scale nonlinear programming. Mathematical Programming 2005; 106(1): 25-57.

32. HSL. A collection of Fortran codes for large scale scientific computation. http: //www.hsl.rl.ac.uk, 2020. (Date accessed: 16.09.2020).

33. Biegler LT, Cervantes AM and Wächter A. Advances in simultaneous strategies for dynamic process optimization. Chemical Engineering Science 2002; 57(4): 575-593.

34. Yi J, Li J, Lu J et al. On the stability and agility of aggressive vehicle maneuvers: A pendulum-turn maneuver example. IEEE Transactions on Control Systems Technology 2012; 20(3): 663676.

35. Huber PJ. Robust estimation of a location parameter. Annals of Mathematical Statistics 1964; 35(1): 73-101.

36. Hartley RI and Zisserman A. Multiple View Geometry in Computer Vision. Second ed. Cambridge, UK: Cambridge University Press, 2004.

37. Fors V. Autonomous Vehicle Maneuvering at the Limit of Friction. Linköping studies in science and technology. Dissertation 2102, Linköping University, Department of Electrical Engineering, 2020. 


\section{Appendix A}

This appendix is a complement to Section "Chassis Model" and gives definitions of the expressions introduced to keep (1)-(5) compact. These definitions are

$$
\begin{aligned}
F_{X}= & F_{x, 1} \cos (\delta)-F_{y, 1} \sin (\delta)+F_{x, 2} \cos (\delta)-F_{y, 2} \sin (\delta)+F_{x, 3}+F_{x, 4}, \\
F_{Y}= & F_{x, 1} \sin (\delta)+F_{y, 1} \cos (\delta)+F_{x, 2} \sin (\delta)+F_{y, 2} \cos (\delta)+F_{y, 3}+F_{y, 4}, \\
M_{Z}= & l_{f}\left(F_{y, 1} \cos (\delta)+F_{y, 2} \cos (\delta)+F_{x, 1} \sin (\delta)+F_{x, 2} \sin (\delta)\right)-l_{r}\left(F_{y, 3}+F_{y, 4}\right) \\
& +w\left(F_{y, 1} \sin (\delta)-F_{y, 2} \sin (\delta)-F_{x, 1} \cos (\delta)+F_{x, 2} \cos (\delta)-F_{x, 3}-F_{x, 4}\right) \\
f_{\theta}(\cdot)= & \sin (\theta) \cos (\phi)\left(\dot{\psi}^{2}+\dot{\phi}^{2}+\dot{\theta}^{2}\right)-\sin (\psi) \ddot{\psi}-2 \cos (\psi) \dot{\phi} \dot{\psi}-\cos (\theta) \cos (\phi) \ddot{\theta} \\
& +2 \cos (\theta) \sin (\phi) \dot{\theta} \dot{\phi}+\sin (\theta) \sin (\phi) \ddot{\phi} \\
f_{\phi}(\cdot)= & -\sin (\theta) \cos (\phi) \ddot{\psi}-\sin (\phi) \dot{\psi}^{2}-2 \cos (\theta) \cos (\phi) \dot{\theta} \dot{\psi} \\
& +\sin (\theta) \sin (\phi) \dot{\phi} \dot{\psi}-\sin (\phi) \dot{\phi}^{2}+\cos (\phi) \ddot{\phi} \\
I_{\psi}(\cdot)= & I_{x x} \sin (\theta)^{2}+\cos (\theta)^{2}\left(I_{y y} \sin (\phi)^{2}+I_{z z} \cos (\phi)^{2}\right) \\
f_{\psi}(\cdot)= & F_{X} \sin (\phi)+F_{Y} \sin (\theta) \cos (\phi) \\
I_{\theta}(\cdot)= & I_{y y} \cos (\phi)^{2}+I_{z z} \sin (\phi)^{2} \\
I_{\phi}(\cdot)= & I_{x x} \cos (\theta)^{2}+I_{y y} \sin (\theta)^{2} \sin (\phi)^{2}+I_{z z} \sin (\theta)^{2} \cos (\phi)^{2}, \\
f_{\theta, 1}= & m g \sin (\theta) \cos (\phi)-F_{X} \cos (\theta) \cos (\phi) \\
f_{\theta, 2}= & -K_{\theta} \theta-D_{\theta} \dot{\theta}+\dot{\psi}\left(\dot{\psi} \sin (\theta) \cos (\theta)\left(I_{x x}-I_{y y}+\cos (\phi)^{2}\left(I_{y y}-I_{z z}\right)\right)\right. \\
& \left.-\dot{\phi}\left(\cos (\theta)^{2} I_{x x}+\sin (\phi)^{2} \sin (\theta)^{2} I_{y y}+\sin (\theta)^{2} \cos (\phi)^{2} I_{z z}\right)-\dot{\theta}\left(\sin (\theta) \sin (\phi) \cos (\phi)\left(I_{y y}-I_{z z}\right)\right)\right) \\
f_{\phi, 1}= & F_{Y} \cos (\phi) \cos (\theta)+m g \sin (\phi) \\
f_{\phi, 2}= & -\left(K_{\phi, f}+K_{\phi, r}\right) \phi-\left(D_{\phi, f}+D_{\phi, r}\right) \dot{\phi}+\dot{\psi}\left(I_{y y}-I_{z z}\right)(\dot{\psi} \sin (\phi) \cos (\phi) \cos (\theta)+\dot{\phi} \sin (\theta) \sin (\phi) \cos (\phi)) \\
& +\dot{\psi} \dot{\theta}\left(\cos (\phi)^{2} I_{y y}+\sin (\phi)^{2} I_{z z}\right)
\end{aligned}
$$

